\title{
Image resonance in the many-body density of states at a metal surface
}

\author{
G. Fratesi* and G. P. Brivio \\ INFM and Dipartimento di Scienza dei Materiali, Università di Milano-Bicocca, via Cozzi 53, 20125 Milano, Italy \\ Patrick Rinke $^{\dagger}$ and R. W. Godby \\ Department of Physics, University of York, York YO10 5DD, United Kingdom
}

(Received 29 May 2003; published 11 November 2003)

\begin{abstract}
The electronic properties of a semi-infinite metal surface without a bulk gap are studied by a formalism that is able to account for the continuous spectrum of the system. The density of states at the surface is calculated within the $G W$ approximation of many-body perturbation theory. We demonstrate the presence of an unoccupied surface resonance peaked at the position of the first image state. The resonance encompasses the whole Rydberg series of image states and cannot be resolved into individual peaks. Its origin is the shift in spectral weight when many-body correlation effects are taken into account.
\end{abstract}

DOI: 10.1103/PhysRevB.68.195404

PACS number(s): 73.20.At, 71.15.-m

\section{INTRODUCTION}

For the understanding of several fundamental properties of condensed-matter surfaces, the knowledge of the electronic density of states and of the parallel-wave-vectorresolved density is essential. The dispersion of both occupied and excited surface states, the influence of the changes of the electronic structure in thin-film growth, and enhanced magnetism are just a few examples of relevant quantities. Experimentally, the density of states can be probed by a variety of techniques such as photoemission spectroscopies ${ }^{1}$ (angleresolved, integrated, inverse, and two-photon) and scanning tunneling microscopy. ${ }^{2}$ To provide an adequate theoretical description of the experimental observables, it is necessary to employ methods which retain the continuous character of the spectrum. In other words, one has to take into account that certain quantities may be defined for any energy in a given energy interval. Furthermore, the formalism must be capable of describing excited-state properties.

Regarding excited states, considerable experimental interest has been devoted to image-potential-induced (IPI) states $^{3-6}$ and resonances. ${ }^{7-10}$ IPI states are present in systems where a bulk band gap provides a barrier, trapping electrons in the image tail of the surface potential. If no gap is present at the IPI energies, an electron is not reflected completely at the bulk barrier, and hybridization with surface truncated bulk states becomes possible. This results in the formation of resonances for some materials. A comprehensive theoretical description of IPI states has been given by Echenique and co-workers, ${ }^{11-14}$ whereas the situation for IPI resonances is much less satisfactory. In principle, IPI resonances are intrinsically contained in the many-body framework, already at the level of the $G W$ approximation, ${ }^{15}$ but to our knowledge surfaces have only been investigated in this context using a repeated slab geometry. ${ }^{16-18}$ Such a simplified treatment cannot capture the continuous spectrum of a real surface, because the spectral function will inevitably be composed of a limited number of sharp, discrete peaks in place of the resonance.

In this paper we present $a b$ initio many-body calculations of the local density of states (LDOS) of a semi-infinite jel- lium surface and demonstrate the presence of a broad IPI resonance. We calculate the LDOS-in its many-body generalization, the spectral function ${ }^{15}$ - decomposed according to the surface parallel wave vector $\mathbf{k}_{\|}$within the surface $x y$ plane as

$$
\sigma(\mathbf{r}, \omega)=\int \frac{\mathrm{d}^{2} \mathbf{k}_{\|}}{(2 \pi)^{2}} A\left(z, \mathbf{k}_{\|}, \omega\right),
$$

where $A$ is the $\mathbf{k}_{\|}$-resolved LDOS or spectral weight function, defined by

$$
A\left(z, \mathbf{k}_{\|}, \omega\right)=-\frac{1}{\pi} \operatorname{Im} G\left(z, z, \mathbf{k}_{\|}, \omega\right) \operatorname{sgn}(\omega-\mu) .
$$

Here $G$ is the one-particle Green's function in the representation indicated, and Hartree atomic units $\left(a_{0}\right.$ $=0.529 \AA, 1$ hartree $=27.2 \mathrm{eV}$ ) are used.

$G$ is obtained from Dyson's equation

$$
G=G^{\mathrm{DFT}}+G^{\mathrm{DFT}}\left[\Sigma_{\mathrm{XC}}-v_{\mathrm{XC}}\right] G,
$$

where $v_{\mathrm{XC}}$ is the exchange and correlation potential in density-functional theory (DFT) and $\Sigma_{\mathrm{XC}}$ is the $G W$ electron self-energy.

Equation (3) is solved using a recent method developed to perform $G W$ calculations in infinite, nonperiodic geometries ${ }^{19}$ based on the embedding method. ${ }^{20}$ The advantage of this approach is that the semi-infinite substrate, surface, and vacuum regions are treated equally without the need for any fitting parameters or a repeated cell geometry.

We recall first the main steps of a $G W$ calculation, given as follows

(i) The Kohn-Sham equation ${ }^{21}$ is solved self-consistently within local-density approximation (LDA), ${ }^{22}$ leading to $G^{\text {DFT }}$.

(ii) From $G^{\mathrm{DFT}}$ the polarization $P$ is computed in the random-phase approximation (RPA).

(iii) The inverse dielectric response $\epsilon^{-1}=(1-v P)^{-1}(v$ is the Coulomb potential) yields the effective interaction $W^{\mathrm{RPA}}=\epsilon^{-1} v$. 


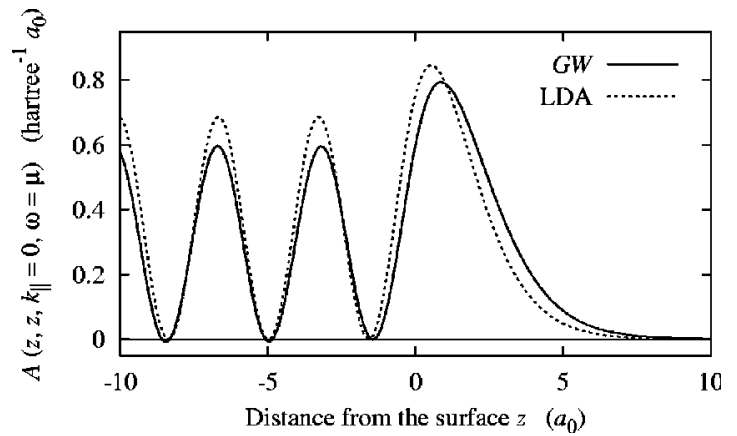

FIG. 1. Spectral weight function at the chemical potential and zero parallel wave vector for semi-infinite jellium with $r_{s}$ $=2.07 a_{0}$.

(iv) The self-energy $\Sigma_{\mathrm{XC}}=i G^{\mathrm{DFT}} W^{\mathrm{RPA}}$ is calculated using a real-space, imaginary-frequency representation, and one obtains the self-energy on the real-frequency axis by means of analytic continuation.

(v) Equation (3) can now be solved to update $G$.

In order to deal with an infinite system, our approach differs from previous works ${ }^{16,17,23}$ based on the supercell geometry. First, $G^{\mathrm{DFT}}$ at stage (i) is obtained by numerical inversion of the Kohn-Sham Hamiltonian in a finite region $\Omega$ comprising the jellium edge, in which the effects of an infinitely extended substrate outside $\Omega$ enter via an extra nonlocal, energy-dependent (embedding) potential only defined at the surface of $\Omega$. Consequently $G^{\mathrm{DFT}}$ describes a truly continuous spectrum of fictitious noninteracting electrons. Second, we exploit the knowledge of the asymptotic dielectric function $\epsilon^{-1}$ both in the solid and in the vacuum to restrict the inversion of $\epsilon$ at stage (iii) to a finite region, whose size plays the role of a convergence parameter. In practice, we increase it until the results match well with the asymptotic ones on the boundaries of such a region.

In Sec. II we present and discuss the results. Section III is devoted to the conclusions.

\section{RESULTS AND DISCUSSION}

We examine first the $z$ dependence of the spectral weight function $A\left(z, \mathbf{k}_{\|}, \omega\right)$. This quantity is proportional to the probability amplitude for a particular wave vector $\mathbf{k}_{\|}$and energy $\omega$. In Fig. 1 we report $A\left(z, \mathbf{k}_{\|}, \omega\right)$ for $k_{\|}$equal to zero and $\omega$ equal to the chemical potential $\mu$.

All results shown in this paper are obtained for a jellium substrate of aluminum density $\left(r_{s}=2.07 a_{0}\right)$. The spectral weight outside the surface is enhanced by the improved description of exchange-correlation effects in $G W$, in common with the states of $\mathrm{Al}(111)$ studied in Ref. 23. By varying the energy $\omega$ we have verified that this feature is common to all bound states. In the bulk, the $G W$ spectral weight is lower than that calculated by the LDA because some weight is transferred to lower energies through electron-plasmon coupling. The larger amplitude of $A$ in the surface layer, together with the absence of decay into the bulk, identifies the states in this part of the surface band structure as forming a surface resonance.

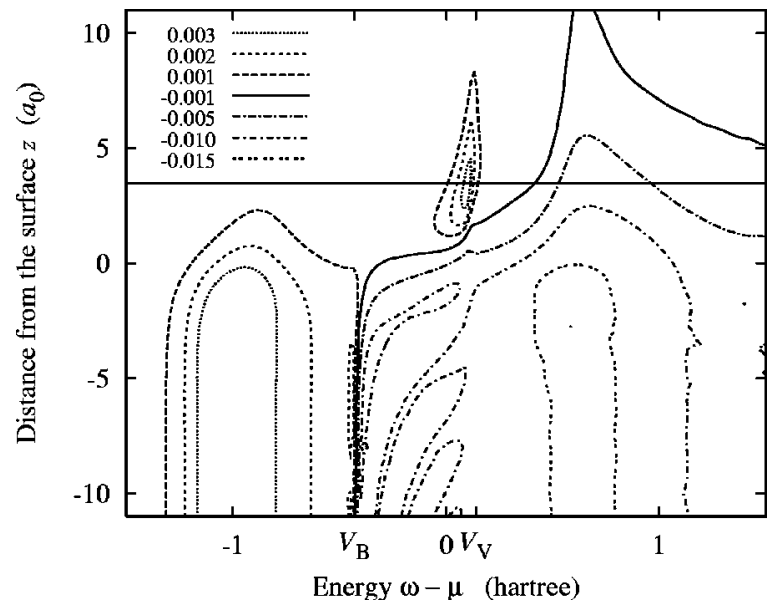

FIG. 2. Contour levels of $\Delta \sigma(z, \omega)$ for semi-infinite jellium with $r_{s}=2.07 a_{0}$. The solid horizontal line indicates $z=3.5 a_{0}$ (see Fig. 4). The pronounced "teardrop" feature at $z \sim 3.5 a_{0}$ is the surface image resonance. $V_{\mathrm{V}}$ is the vacuum energy level, and $V_{\mathrm{B}}$ the bottom of the free-electron band.

In Fig. 2 we plot $\Delta \sigma=\sigma^{G W}-\sigma^{\mathrm{LDA}}$, the difference between our many-body LDOS and that in the LDA.

The energy dependence of $\Delta \sigma$ as $z$ moves from the bulk towards the vacuum is indicated by contour levels. We recapitulate that, for a jellium surface, the LDA effective potential $v_{\text {eff }}(z)$ approaches the constant limits $V_{\mathrm{B}}$ and $V_{\mathrm{V}}$ for $z$ $\rightarrow-\infty$ (bulk) and $z \rightarrow+\infty$ (vacuum), respectively. As a consequence, the bulk and vacuum limits of $\sigma^{\mathrm{LDA}}$ are simply proportional to the well-known expressions $\sqrt{\omega-V_{\mathrm{B}}}$ and $\sqrt{\omega-V_{\mathrm{V}}}$. In the bulk, the LDA therefore predicts no states below $V_{\mathrm{B}}$. The electron-plasmon coupling, automatically included in $G W$, is responsible for moving states down in energy from above to below $V_{\mathrm{B}}$, yielding the subband shown in Fig. 3 for energies around 1 hartree below the chemical potential, as already demonstrated for the homogeneous electron gas. ${ }^{15}$

The presence of the surface is still noticeable even some atomic units into the substrate through Friedel oscillations, which are visible for bound energies from the contour levels of Fig. 2 and in the LDOS at a distance from the surface $z$ $=-10 a_{0}$ in Fig. 3 .

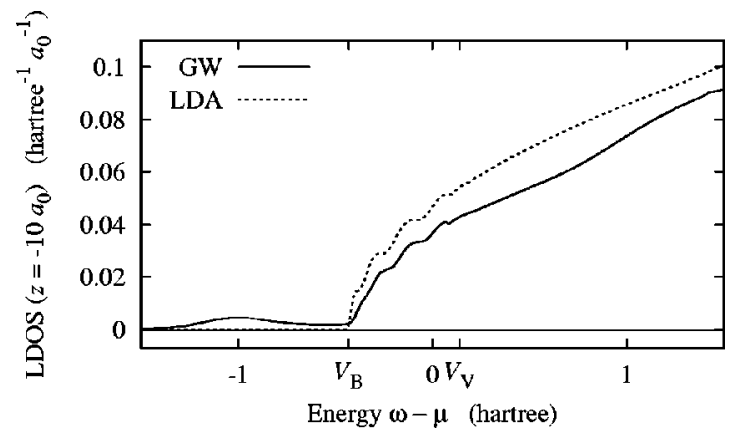

FIG. 3. LDOS $\sigma^{G W}$ and $\sigma^{\mathrm{LDA}}$ for semi-infinite jellium with $r_{s}$ $=2.07 a_{0}$ at $z=-10 a_{0}$ from the jellium edge. The "ripples" between $V_{\mathrm{V}}$ and $V_{\mathrm{B}}$ indicate the presence of Friedel oscillations. 


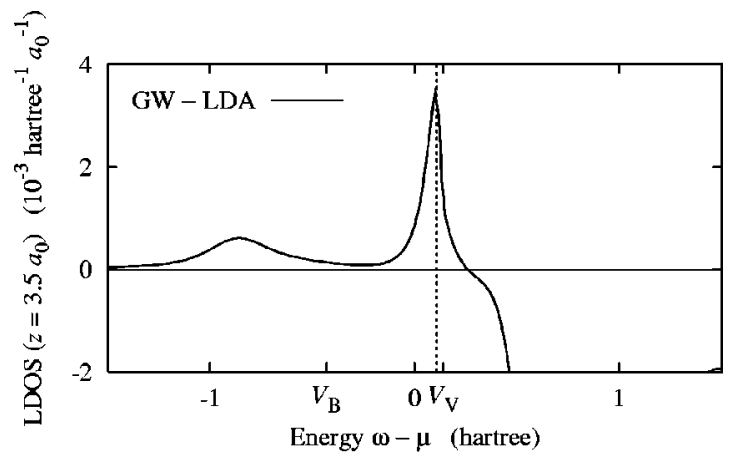

FIG. 4. Difference in the LDOS $\Delta \sigma$ for semi-infinite jellium with $r_{s}=2.07 a_{0}$ at $z=3.5 a_{0}$ from the surface. The dashed vertical line indicates the position of the first Rydberg state, 1/32 hartree below the vacuum level.

When $z$ approaches the surface, coupling with plasmons considerably reduces, eventually becoming negligible a few atomic units outside the surface. As $z$ moves into the vacuum, the electron density decays to zero. Self-energy effects are no longer present and the LDA description becomes exact. We then obtain $\Delta \sigma=0$.

Apart from the surface-truncated bulk structure, Fig. 2 also highlights a completely new feature in the form of a teardrop-shaped enhancement of the LDOS, localized in the near-surface region at energies between the chemical potential and the vacuum level. In order to examine this feature in more detail we plot the energy dependence of $\Delta \sigma$ for $z$ $=3.5 a_{0}$ in Fig. 4 (note that this corresponds to a crosssection plot along a horizontal line through Fig. 2).

The peak in $\Delta \sigma$, located at about $1 / 32$ hartree below the vacuum level (the position of the first Rydberg state ${ }^{11}$ ), clearly identifies an IPI surface resonance.

The presence of IPI resonances, even in the limiting case of a substrate without bulk reflectivity (the bulk reflectivity is associated with scattering by the atomic $\operatorname{cores}^{24}$ ), has been unclear. Simple model potentials in independent-particle approximations produced IPI resonances for jellium substrates $^{25}$ in some cases, but the presence of a clear resonance depended sensitively on the precise details of the chosen model. We recall that IPI states and resonances are a many-body phenomenon and can thus be included only in an ad hoc way in single-particle dynamic approximations. In contrast to these earlier, parameter-dependent results, our method is fully $a b$ initio and includes many-body correlations.

We also remark that single-particle calculations, even if using the "true" DFT effective potential (e.g., the one computed from $\Sigma_{\mathrm{XC}}$ in Refs. 16 and 17) cannot access properly the quasiparticle spectral properties of the system. ${ }^{29}$ In the many-body formalism adopted in this paper the quasiparticle spectrum and amplitudes are instead automatically included in the interacting Green's function $G$. The interaction with the image charge is implicitly contained in the self-energy since the $G W$ approximation accounts for its physical origin, i.e., the electron-plasmon coupling. ${ }^{26}$

In the case of semi-infinite jellium, the zero bulk reflec-

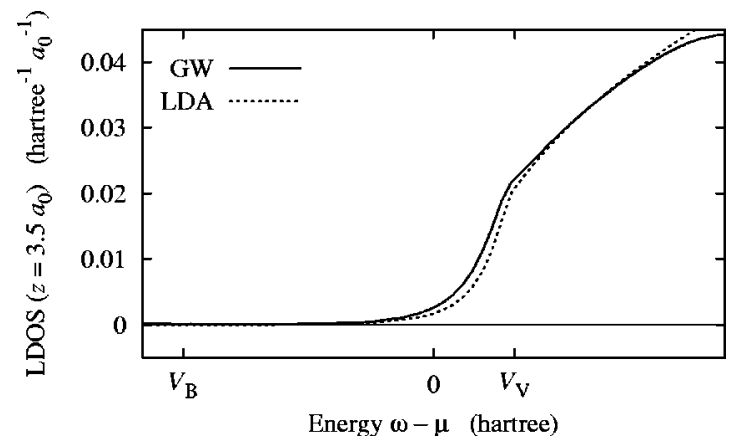

FIG. 5. Local density of states for a semi-infinite jellium with $r_{s}=2.07 a_{0}$ at $z=3.5 a_{0}$. The quantity reported in Fig. 4 is the difference between the continuous and the dashed line.

tivity yields a large linewidth of the resonance, since hybridization with bulk states is not prevented at all. We estimate the full width at half maximum (FWHM) from $\Delta \sigma$ as 0.07 hartree (about $2 \mathrm{eV}),{ }^{30}$ and remark that in systems without a bulk band gap the hybridization with the continuous bulk band is the major contributor to the linewidth, as the selfenergy alone accounts only for about one-tenth of the total FWHM. $^{14}$

Owing to its large linewidth, the resonance shown in Fig. 4 encompasses the whole Rydberg series of image states, ${ }^{8}$ which in the infinite bulk barrier model extends from $V_{\mathrm{V}}$ $-1 / 32$ hartree to $V_{\mathrm{V}}$. The resonance is clearly visible in the difference plot between the many-body LDOS and the LDOS in the LDA. In the many-body LDOS itself, on the other hand, it resolves as a shoulder feature just below the vacuum energy (Fig. 5) rather than a distinct peak, because the LDA LDOS is already a strongly increasing function of energy in this range.

The quantity reported in Fig. 4 is the difference between two theoretical quantities and is therefore not directly accessible to experiment. (The measured spectra will also depend on matrix elements and hence on the specific experimental technique used and on a more realistic description of the substrate under investigation.) However, it allows us to isolate an important contribution to the phenomenon-the many-body interaction-which could improve the description of the electronic structure of real semi-infinite surfaces beyond the usual single-particle approaches such as Ref. 27.

We will now examine the origin of the resonance emerging in Fig. 4. For this reason it is important to look at the spectral weight function $A$, whose integral over $\mathbf{k}_{\|}$is the LDOS [Eq. (1)]. In Fig. 6 we present the energy dependence of $A\left(z, \mathbf{k}_{\|}, \omega\right)$ for $\mathbf{k}_{\|}=0$ at the same position outside the jellium edge as Figs. 4 and $5\left(z=3.5 a_{0}\right)$.

We first consider the LDA result. For noninteracting particles in a constant potential $V$ the $z$-resolved spectral weight function is essentially onedimensional and hence proportional to $\left(\omega-k_{\|}^{2} / 2-V\right)^{-1 / 2}$. Thus in the vacuum a singularity is present at $V_{\mathrm{V}}$ for $k_{\|}=0$. The spectral weight then diminishes with increasing energy. But near the surface weight is transferred to bulk states decaying out into the vacuum: the spectral function has a peak very close to $v_{\text {eff }}(z)$, but singularities are no longer present. We emphasize that in a slab 


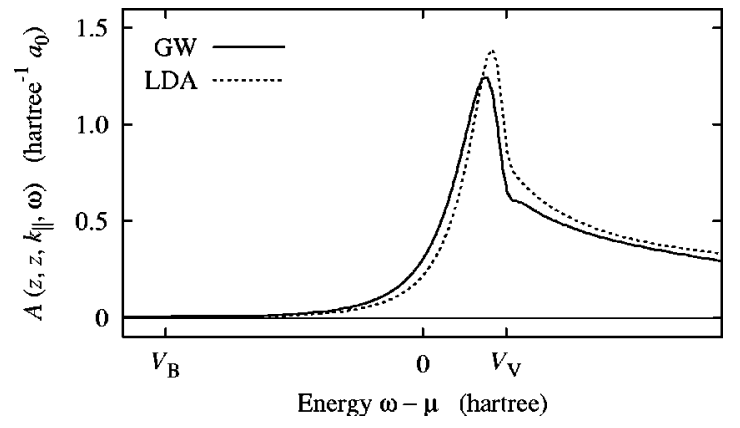

FIG. 6. Spectral weight function for a semi-infinite jellium with $r_{s}=2.07 a_{0}$ for $k_{\|}=0$ and $z=3.5 a_{0}$. The integral over $\mathbf{k}_{\|}$of this function gives the LDOS reported in Fig. 5.

geometry Fig. 6 would appear as a collection of $\delta$ functions, each $\delta$ corresponding to one of the discrete eigenstates.

When we describe the system in the interacting picture $(G W)$, we still observe bulk states that spill out into the vacuum. But we also have a new class of states, constituting the IPI resonance. They have substantial weight at and outside the surface. As a consequence spectral weight from the LDA states is transferred into these quasiparticle states. Spectral weight hence moves down from higher energies to the energies of the IPI resonance. This produces the shoulder in $A$ at $V_{\mathrm{V}}$ and the displacement of the peak energy to lower energies.

If $k_{\|}$is increased from its zero value, the profile of $A$ shown in Fig. 6 is shifted towards higher energies by $k_{\|}^{2} / 2$, without being distorted too much (i.e., the dispersion of the states is nearly parabolic with effective mass equal to 1). When we integrate over $k_{\|}$to get the LDOS, one notices that for energies roughly below $V_{\mathrm{V}}$ the $G W$ spectral weight $A^{G W}$ is greater than $A^{\mathrm{LDA}}$ for any value of $k_{\|}$, leading to the positive $\Delta \sigma$ shown in Fig. 4. For higher energies, $A^{G W}$ $-A^{\text {LDA }}$ changes from negative to positive as $k_{\|}$increases. Negative contributions dominate and the corresponding $\Delta \sigma$ is negative.

\section{CONCLUSIONS}

In summary, we have evaluated the local density of states of a semi-infinite simple metal surface from first principles. Many-body correlations are included via the $G W$ selfenergy. The substrate is described as truly semi-infinite, thus enabling a calculation of the continuous spectrum necessary to properly account for the hybridization between surface electronic states and bulk states. We demonstrate the presence of an IPI resonance just below the vacuum energy, encompassing the full series of image states that would be present in a system with a surface band gap. The origin of the resonance is explained in terms of the spectral weight transfer to lower energies due to the inclusion of electronelectron correlation.

\section{ACKNOWLEDGMENTS}

We are grateful to S. Modesti, L. G. Molinari, G. Onida, and M. I. Trioni for useful discussions. This work was supported by the Italian MIUR through Grant No. 2001021128. P.R. acknowledges the support of EPSRC and the DAAD.
*Present address: SISSA/ISAS, via Beirut 4, 34014 Trieste, Italy.

†Present address: Fritz-Haber-Institut der Max-Planck-Gesellschaft, Faradayweg 4-6, 14195 Berlin-Dahlem, Germany.

${ }^{1}$ R. Matzdorf, Surf. Sci. Rep. 30, 153 (1998).

${ }^{2}$ S. Schintke, S. Messerli, M. Pivetta, F. Patthey, L. Libioulle, M. Stengel, A.D. Vita, and W.-D. Schneider, Phys. Rev. Lett. 87, 276801 (2001).

${ }^{3}$ V. Dose, W. Altmann, A. Goldmann, U. Kolak, and J. Rogozik, Phys. Rev. Lett. 52, 1919 (1984).

${ }^{4}$ D. Straub and F.J. Himpsel, Phys. Rev. Lett. 52, 1922 (1984).

${ }^{5}$ K. Giesen, F. Hage, F.J. Himpsel, H.J. Riess, and W. Steinmann, Phys. Rev. Lett. 55, 300 (1985).

${ }^{6}$ T. Hertel, E. Knoesel, M. Wolf, and G. Ertl, Phys. Rev. Lett. 76, 535 (1996).

${ }^{7}$ D. Straub and F.J. Himpsel, Phys. Rev. B 33, 2256 (1986).

${ }^{8}$ B. Quiniou, V. Bulvović, and R.M. Osgood, Phys. Rev. B 47, 15890 (1993).

${ }^{9}$ S. Yang, R.A. Bartynski, G.P. Kochanski, S. Papadia, T. Fondén, and M. Persson, Phys. Rev. Lett. 70, 849 (1993).

${ }^{10}$ L. Petaccia, L. Grill, M. Zangrando, and S. Modesti, Phys. Rev. Lett. 82, 386 (1999).

${ }^{11}$ P.M. Echenique and J.B. Pendry, J. Phys. C 11, 2065 (1978).

${ }^{12}$ P.M. Echenique, F. Flores, and F. Sols, Phys. Rev. Lett. 55, 2348 (1985)

${ }^{13}$ J. Bausells and P.M. Echenique, Phys. Rev. B 33, 1471 (1986).
${ }^{14}$ E.V. Chulkov, I. Sarría, V.M. Silkin, J.M. Pitarke, and P.M. Echenique, Phys. Rev. Lett. 80, 4947 (1998).

${ }^{15}$ L. Hedin and S. Lundqvist, Solid State Physics (Academic Press, New York, 1969), Vol. 23.

${ }^{16}$ A.G. Eguiluz, M. Heinrichsmeier, A. Fleszar, and W. Hanke, Phys. Rev. Lett. 68, 1359 (1992).

${ }^{17}$ J.J. Deisz, A.G. Eguiluz, and W. Hanke, Phys. Rev. Lett. 71, 2793 (1993).

${ }^{18}$ M. Heinrichsmeier, A. Fleszar, W. Hanke, and A.G. Eguiluz, Phys. Rev. B 57, 14974 (1998).

${ }^{19}$ G. Fratesi, G. P. Brivio, and L. G. Molinari, cond-mat/0305344 (unpublished).

${ }^{20}$ J.E. Inglesfield, J. Phys. C 14, 3795 (1981).

${ }^{21}$ W. Kohn and L.J. Sham, Phys. Rev. 140, A1133 (1965).

${ }^{22}$ J.P. Perdew and A. Zunger, Phys. Rev. B 23, 5048 (1981).

${ }^{23}$ I.D. White, R.W. Godby, M.M. Rieger, and R.J. Needs, Phys. Rev. Lett. 80, 4265 (1998).

${ }^{24}$ S. Papadia, M. Persson, and L.-A. Salmi, Phys. Rev. B 41, 10237 (1990).

${ }^{25}$ S.A. Lindgren and L. Walldén, Phys. Rev. B 40, 11546 (1989).

${ }^{26}$ E. Evans and D.L. Mills, Phys. Rev. B 8, 4004 (1973).

${ }^{27}$ H. Ishida, Phys. Rev. B 63, 165409 (2001).

${ }^{28}$ V. Bulović, B. Quiniou, and R.M. Osgood, J. Vac. Sci. Technol. A 12, 2201 (1994).

${ }^{29}$ For example, it has proven impossible to construct a complex 
effective potential which gives the correct damping for all the states of a jellium surface (Ref. 17).

${ }^{30}$ Experimentally, the FWHMs of surface resonances at metal surfaces are usually of the order of tenths of eV (Refs. 8 and
28). The difference may be explained by the non-negligible reflectivity of the atomic cores, reducing the hybridization with the underlying bulk continuum of states and thus the FWHM. 